\title{
Impact of Pupil Aberrations on Wavefront Manipulation
}

\author{
Thomas Nobis ${ }^{1, *}$ \\ ${ }^{1}$ Carl Zeiss AG, Corporate Research and Technology, Jena location, Carl-Zeiss-Promenade 10, 07745 Jena, Germany
}

\begin{abstract}
A systematic and quantitative analysis is given of the impact of pupil aberrations on the imaging performance in wavefront manipulation applications using adaptive optical elements. For the practical case of rotationally-symmetric types of wavefront corrections, such as defocus or spherical aberration, analytical expressions of the induced aberrations are derived including their pupil and field dependence. Each aberration is thereby related to the specific pupil aberration present at the adaptive element. The results can be used to specify the acceptable amount of pupil correction required for a specific magnitude and type of wavefront manipulation.
\end{abstract}

\section{Introduction}

In the recent decade, the technology of adaptive optical elements, such as spatial light modulators or deformable mirrors, has rapidly improved. Wavefront manipulation is an obvious application of these devices, where they, e.g., either generate or compensate for a specific phase profile in the imaging path. Especially in wide-field imaging, the adaptive element is typically placed at a conjugated pupil plane. The footprints of all ray bundles are identical there, and all field points are therefore affected in the same manner by the element.

For such application, the considered optical system must provide an accessible pupil plane. Depending on the specific application, either the image of the entrance pupil or the image of the exit pupil onto that element must reach a certain level of correction. In practical cases, however, the intermediate image of the pupil is not ideal, i.e., pupil aberrations are present. They can be classified in the same systematic way as aberrations in the image plane, as well as there are analytical Seidel surface contributions based on paraxial ray data similar as for the common imaging aberrations [1]. Usually, investigations of pupil aberrations aim for their direct relation to image correction. Doubtless, the most-famous case is the Abbe sine condition, a specific type of pupil distortion, and its relation to coma correction for object imaging [2]. Other known influences of pupil aberrations are, e.g., beam deformations [1], changes in relative illumination [3] or additional aberrations when conjugates are changed [4-6]. The occurrence of field-dependent aberrations caused by pupil aberrations in adaptive optics is also known [7,8]. However, a systematic and quantitative approach is still missing, describing both the induced types of image aberrations and the acceptable amount of pupil aberrations depending on the specific wavefront manipulation being done. In this paper, such systematic overview and a quantitative analysis is given of the impact of pupil aberrations on wavefront manipulation with adaptive phase elements.

\section{Theory}

Aberrations of rotational-symmetric optical systems can be expressed by the well-known Seidel series both as wavefront deviations $W$ from the reference sphere in the exit pupil as well and transverse ray aberrations $\vec{T}$ from the ideal image point in the image plane. Therefore, a ray is considered starting in the object plane at object height $h$ and hitting the entrance pupil at a radius $r$ at an angle $\vartheta$ with respect to the meridional plane, as given in Fig. 1. The wavefront aberration, $W$, then reads [2]

$$
\begin{aligned}
& W(h, r, \vartheta)=\underbrace{W_{020} r^{2}}_{\text {Defocus }}+\underbrace{W_{111} h r \cos \vartheta}_{\text {Magnification }}+ \\
& \underbrace{W_{040} r^{4}}_{\text {Spherical Ab. }}+\underbrace{W_{131} h r^{3} \cos \vartheta}_{\text {Coma }}+\underbrace{W_{220} h^{2} r^{2}}_{\text {Sagittal field curv. }}+ \\
& \underbrace{W_{222} h^{2} r^{2} \cos ^{2} \vartheta}_{\text {Astigmatism }}+\underbrace{W_{311} h^{3} r \cos \vartheta}_{\text {Distortion }}+[\ldots],
\end{aligned}
$$

where the coefficients $W_{k l m}$ are a measure of the overall amount of the specific aberration present. The curly brackets indicate the common names for the two first-order terms and the five third-order Seidel aberrations. Higher-order terms are not regarded, here.

For wavefront manipulation a thin phase element is considered, placed at an intermediate conjugated pupil plane, see Fig. 1. The phase element is supposed to add a specific optical phase $M(\vec{R})$ to the total optical path of each ray, depending on the position $\vec{R}$ where the ray hits the adaptive element assuming that the entrance pupil is imaged perfectly onto the adaptive element. $|M|$ is considered to be small, such that the overall ray path after the element is unchanged and no further induced aberrations are generated.

\footnotetext{
* Corresponding author: thomas.nobis@zeiss.com
} 


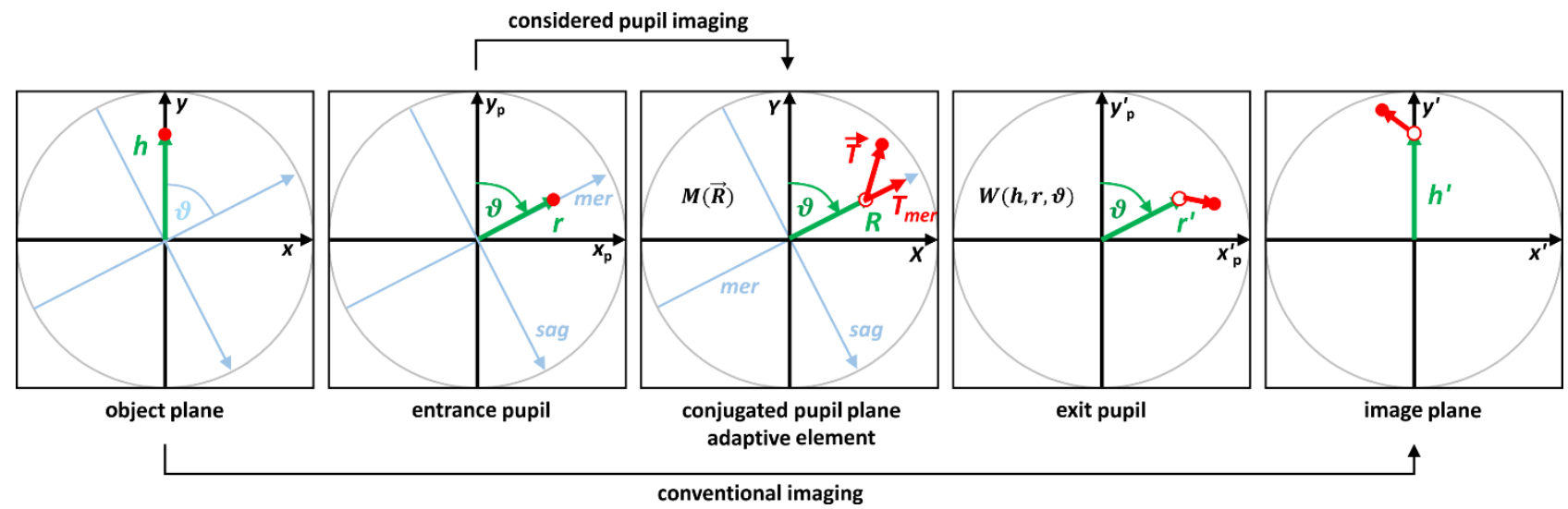

Fig. 1. Coordinate systems for object, pupil and image planes of a rotationally-symmetric optical system. Positions, where a selected ray hits the planes, are marked with a red dot, respectively. A solid dot gives the actual position, while a blank dot indicates the ideal position with no aberrations present. The vector between these dots yields the transverse ray aberration in that plane. The green vector indicates the coordinates of the ideal ray location in polar coordinates in each plane. For object imaging, the line connecting the optical axis with the object point determines the meridional direction by definition, which is the y-direction here. For pupil imaging, however, object and pupil planes exchange their role [1]. Therefore, the meridional direction for the Seidel series of pupil aberrations is determined in the entrance pupil plane by the line between the optical axis and the intersection of the ray in that plane. This direction together with the corresponding sagittal direction is indicated by the light-gray coordinate system.

However, pupil aberrations may lead to a transverse aberration $\vec{T}$ of the considered ray. Hence, an additional contribution $\Delta W$ is added to the wavefront, which is considered as an induced aberration for object imaging. If $|\vec{T}|$ is small, $\Delta W$ can be approximated by the first-order term of a Taylor series of $M$,

$$
\Delta W=M(\vec{R}+\vec{T})-M(\vec{R}) \approx \operatorname{grad}(M) \cdot \vec{T}
$$

i.e. as the scalar product of the gradient of $M$ and $\vec{T}$. This already gives a first quantitative estimation of the maximum allowed $|\vec{T}|$, since $\operatorname{grad}(M)$ is known and the maximum acceptable $\Delta W$ is typically specified for object imaging. For further systematic investigation we restrict ourselves to rotationally-symmetric wavefront corrections, i.e.

$$
M(\vec{R})=M(R)=M_{2} \cdot R^{2}+M_{4} \cdot R^{4}+[\ldots] .
$$

Such restriction is necessary to not break the rotational symmetry of the overall system. In this case, $\operatorname{grad}(M)$ only has a radial component

$$
\operatorname{grad}(M)=\frac{\partial M}{\partial R} \cdot \overrightarrow{e_{R}},
$$

and the scalar product yields the radial component of $\vec{T}$. From the viewpoint of pupil imaging, this is actually the meridional component of $\vec{T}$, i.e. $T_{\text {mer }}$,

$$
\Delta W \cong \operatorname{grad}(M) \cdot \vec{T}=\frac{\partial M}{\partial R} \cdot T_{\mathrm{mer}},
$$

as indicated in Fig. 1.

$T_{\text {mer }}$ can be expressed as a Seidel series similar to $W$, but for transverse aberrations, as e.g. in [9]. For the series expansion the identical ray is used as for $W$. Since we are now considering pupil imaging, however, the object plane and entrance pupil plane exchange their roles [1], and $r$ now stands for the object height, while $h$ refers the pupil radial coordinate. The series up to third-order aberrations [9] then translates into

$$
\begin{gathered}
T_{\text {mer }}=\underbrace{\sigma_{0} \cdot h \cos \vartheta}_{\text {Defokus }}+\underbrace{\sigma_{0}^{\prime} \cdot r}_{\text {Magnification }}+ \\
\underbrace{\sigma_{1} \cdot h^{3} \cos \vartheta}_{\text {Spherical Ab. }}+\underbrace{\sigma_{2} \cdot\left(1+2 \cos ^{2} \vartheta\right) \cdot h^{2} r}_{\text {Coma }}+ \\
\underbrace{\left(3 \sigma_{3}+\sigma_{4}\right) \cdot h \cos \vartheta r^{2}}_{\text {Astigmatism \& Field Curv. }}+\underbrace{\sigma_{5} \cdot r^{3}}_{\text {Distortion }}+[\ldots] .
\end{gathered}
$$

Again, the common names of the terms are indicated by the curly brackets. The coefficients $\sigma_{1 . .5}$ are the wellknown Seidel coefficients. The coefficients $\sigma_{0}$ and $\sigma_{0}^{\prime}$ refer to the respective coefficient for first-order aberrations.

Eqn. (5) now turns out as a product of two polynomial series. The systematic expansion into $6 \times 2$ terms is given in Table 1 . The radius $R$ is substituted by $r$ using the paraxial pupil magnification $\beta$ between the entrance pupil and the adaptive element, i.e. $R=\beta r$. By comparison with Eqn. (1), each term identifies a specific type of wavefront aberration. The quantitative amount $W_{k l m}$ of the induced aberration is given by the respective prefactor in each cell. Table 1 therefore gives the systematic impact of pupil aberrations in wavefront manipulation applications. If, e.g., the adaptive element is used for defocus compensation ( $1^{\text {st }}$ column), and pupil imaging suffers from coma ( $4^{\text {th }}$ line), object imaging will be affected by sagittal field curvature and astigmatism. Higher order corrections or pupil aberrations can be obtained in a similar way.

\section{Example and Discussion}

An example optical system demonstrating the discussed effects of pupil aberrations is given in Fig. 2. The system in Fig. 2(a) is a symmetrical relay lens imaging an object field diameter of $10 \mathrm{~mm}$ onto an image diameter of the same size.

* Corresponding author: thomas.nobis@zeiss.com 
Table 1. Systematic influence of pupil aberrations on the effect of wavefront manipulation.

\begin{tabular}{|c|c|c|}
\hline & \multicolumn{2}{|c|}{ Resulting induced wavefront aberration for object imaging while doing (a) or (b) } \\
\hline $\begin{array}{l}\text { Pupil transverse } \\
\text { aberration }\end{array}$ & $\begin{array}{l}\text { (a) focus correction } \\
\partial M / \partial R=2 M_{2} \cdot R\end{array}$ & $\begin{array}{c}\text { (b) correction of low-order spherical } \\
\text { aberration } \\
\partial M / \partial R=4 M_{4} \cdot R^{3}\end{array}$ \\
\hline$\underbrace{\sigma_{0} \cdot h \cos \vartheta}_{\text {Defokus }}$ & $2 M_{2} \beta \cdot \sigma_{0} \cdot \underbrace{h r \cos \vartheta}_{\text {Magnification }}$ & $4 M_{4} \beta^{3} \cdot \sigma_{0} \cdot \underbrace{h r^{3} \cos \vartheta}_{\text {Coma }}$ \\
\hline$\underbrace{\sigma_{0}^{\prime} \cdot r}_{\text {Magnification }}$ & $2 M_{2} \beta \cdot \sigma_{0}^{\prime} \cdot \underbrace{r^{2}}_{\text {Defocus }}$ & $4 M_{4} \beta^{3} \cdot \sigma_{0}^{\prime} \cdot \underbrace{r^{4}}_{\text {Spherical Aberration }}$ \\
\hline$\underbrace{\sigma_{1} \cdot h^{3} \cos \vartheta}_{\text {Spherical Ab. }}$ & $2 M_{2} \beta \cdot \sigma_{1} \cdot \underbrace{h^{3} r \cos \vartheta}_{\text {Distortion }}$ & $4 M_{4} \beta^{3} \cdot \sigma_{1} \cdot \underbrace{h^{3} r^{3} \cos \vartheta}_{\text {Field-cubic coma }}$ \\
\hline$\underbrace{\sigma_{2} \cdot\left(1+2 \cos ^{2} \vartheta\right) \cdot h^{2} r}_{\text {Coma }}$ & $2 M_{2} \beta \cdot \sigma_{2} \cdot \underbrace{\left(1+2 \cos ^{2} \vartheta\right) \cdot h^{2} r^{2}}_{\text {Sagital field curv. + Astigmatism }}$ & $\begin{array}{l}4 M_{4} \beta^{3} \cdot \sigma_{2} \\
\cdot \underbrace{\left(1+2 \cos ^{2} \vartheta\right) \cdot h^{2} r^{4}}_{\text {Spherical Ab. \& higher-order astigmatism }}\end{array}$ \\
\hline$\underbrace{\left(3 \sigma_{3}+\sigma_{4}\right) \cdot h \cos \vartheta r^{2}}_{\text {Astigmatism \& Field Curv. }}$ & $2 M_{2} \beta \cdot\left(3 \sigma_{3}+\sigma_{4}\right) \cdot \underbrace{h r^{3} \cos \vartheta}_{\text {Coma }}$ & $4 M_{4} \beta^{3} \cdot\left(3 \sigma_{3}+\sigma_{4}\right) \cdot \underbrace{h r^{5} \cos \vartheta}_{\text {5th order coma }}$ \\
\hline$\underbrace{\sigma_{5} \cdot r^{3}}_{\text {Distortion }}$ & $2 M_{2} \beta \cdot \sigma_{5} \cdot \underbrace{r^{4}}_{\text {Spherical Aberration }}$ & $4 M_{4} \beta^{3} \cdot \sigma_{5} \cdot \underbrace{r^{6}}_{\text {5th order spherical aberration }}$ \\
\hline
\end{tabular}
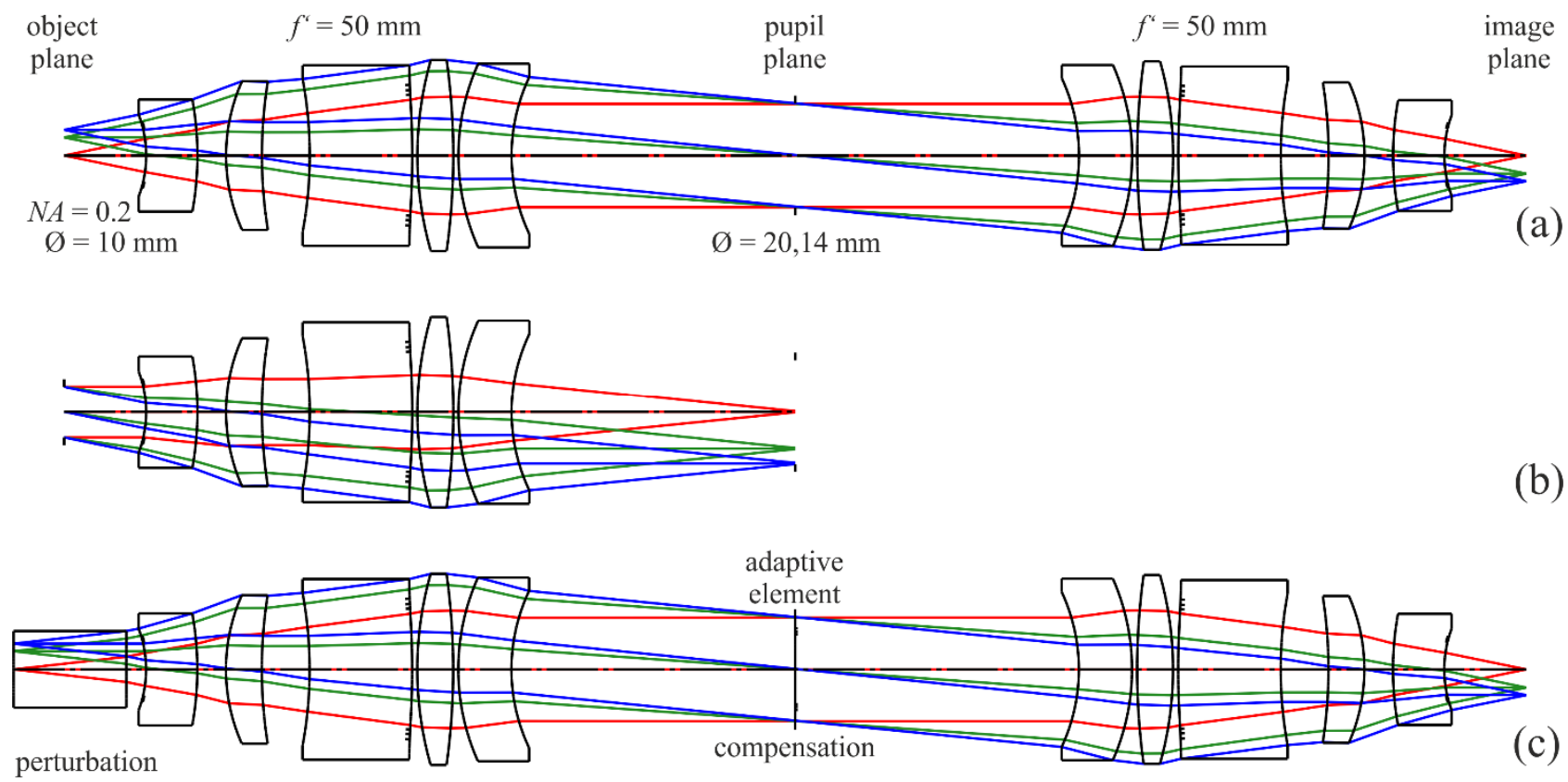

Fig. 2. Example system. (a) Relay system in its initial symmetric state without adaptive element. (b) Imaging of the entrance pupil at infinity to the conjugated pupil plane in the center of the relay system, where the adaptive element can be placed. (c) Relay system in its perturbed state with a glass plate inserted in object space. A thin adaptive element is placed at the conjugated pupil plane and compensates for the $3^{\text {rd }}$-order spherical aberration introduced by the glass plate. Different colors indicate different object points. Onaxis object or pupil points are in red, zonal object or pupil points are in green, and edge points are in blue.

The focal length of the front and back group is $50 \mathrm{~mm}$, and the object and image space numerical aperture is 0.2 , respectively. While the entrance and exit pupil are each located at infinity, a conjugated pupil plane is right in the center of the relay, where a thin adaptive phase element is placed as given in Fig. 2(c). Pupil imaging from the entrance pupil to the conjugated pupil plane is given in Fig. 2(b). The system is corrected monochromatically for a wavelength of $550 \mathrm{~nm}$. It consists of $2 \times 5$ lenses made of the Schott glass N-LASF44 with refractive index $n=$ 1.8079 for the considered wavelength. The first and the

*Corresponding author: thomas.nobis@zeiss.com 
sixth surface of the front group as well as their symmetrical counterparts in the rear group are aspherical, while all other surfaces are spherical. The system is considered as a pure example for the given theory and is specifically prepared to pronounce a selected type of pupil aberration. It has, however, no further application nor has it been manufactured. The performance of the system for object imaging in the initial state is given in Fig. 3 in terms of wavefront aberrations for three field heights. Strehl ratio for these field points are 0.999, 0.989 and 0.970 , respectively, indicating that the system is highly diffracttion-limited over the whole field of view.

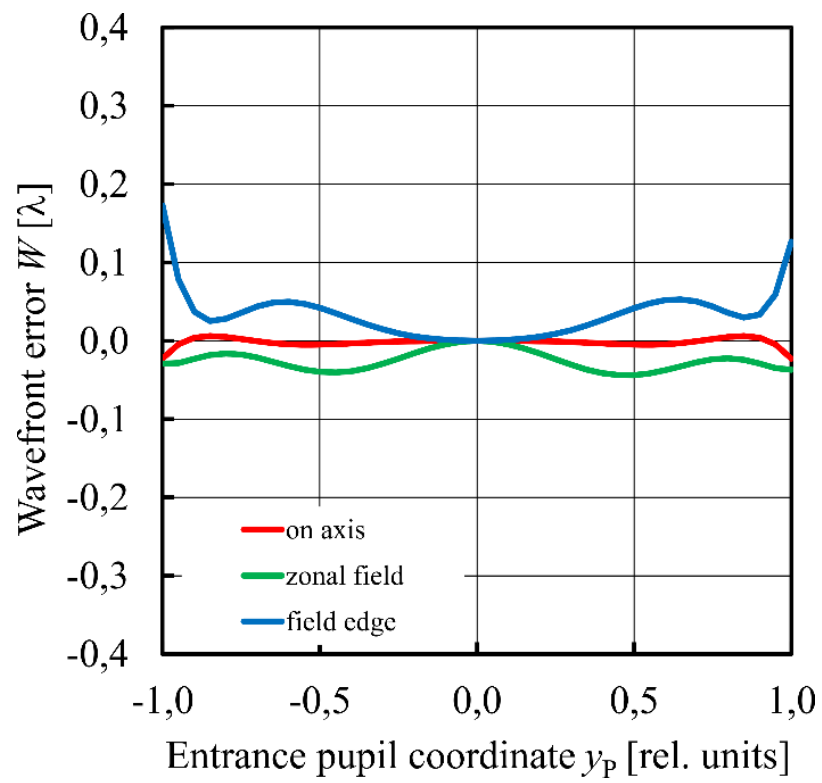

Fig. 3. Wavefront aberration $W$ in meridional direction $(\vartheta=0)$ of the initial system given in Fig. 2(a) for three object heights $h=0.0 \mathrm{~mm}$ (on axis, red), $h=3.54 \mathrm{~mm}$ (zonal, green) and $h=$ $5.0 \mathrm{~mm}$ (field edge, blue).

Further efforts, however, have been made to pronounce a specific type of pupil aberration while suppressing all others. The transverse aberration diagram for pupil imaging as shown in Fig. 2(b) is given in Fig. 4 for three pupil heights. As can be seen, all pupil points suffer from the same amount of $3^{\text {rd }}$-order spherical aberration

$$
T_{\text {mer }}=\sigma_{1} \cdot h^{3} \cos \vartheta=T_{\max } \cdot \frac{h^{3}}{h_{\max }^{3}} \cos \vartheta
$$

with $T_{\max }=-116,95 \mu \mathrm{m}$ and $h_{\max }=5 \mathrm{~mm}$. The differences between the three curves are below $1 \mu \mathrm{m}$, and therefore two orders of magnitude lower. Note that, however, the actual diameter of the conjugated pupil plane is $20.14 \mathrm{~mm}$. This is neither $20.41 \mathrm{~mm}$, as it would be the case for zero tangent distortion in pupil imaging, nor is it $20.00 \mathrm{~mm}=50 \mathrm{~mm} \times 0.2 \times 2$, as it would be the case if the front group was corrected for coma in object imaging, and the sine condition was fulfilled in the conjugated pupil plane. The effects of the residual tangent distortion of $-1.3 \%$ in that plane are discussed below.

The system of Fig. 2(a) is perturbed by inserting a glass plate in object space made of NLASF44 with a thickness of $22 \mathrm{~mm}$, as shown in Fig. 2(c). The system has then been refocused to the paraxial image plane by adjusting the working distance in object space. Hence, from a paraxial point of view, the ray heights and ray angles of the perturbed system are identical to the one in the initial state, and so are the intrinsic Seidel contributions to object imaging. However, as a result of the inserted glass block, a specific amount of mainly $3^{\text {rd }}$ order spherical aberration is introduced to all ray bundles for object imaging. This specific aberration is compensated by a thin phase element adding a positive amount of spherical aberration

$$
M=M_{4} \cdot R^{4}=M_{\max } \cdot \frac{R^{4}}{R_{\max }^{4}}
$$

with $M_{\max }=+3.16 \lambda$ and $R_{\max }=10,07 \mathrm{~mm}$ to the wavefront. The specific value of $M_{\max }$ was determined numerically by restoring the best-focus on axis in image space using optical design software, as it would be the case for an adaptive optics setup. Without this compensation, the diffraction-limited performance of the system would be completely gone.

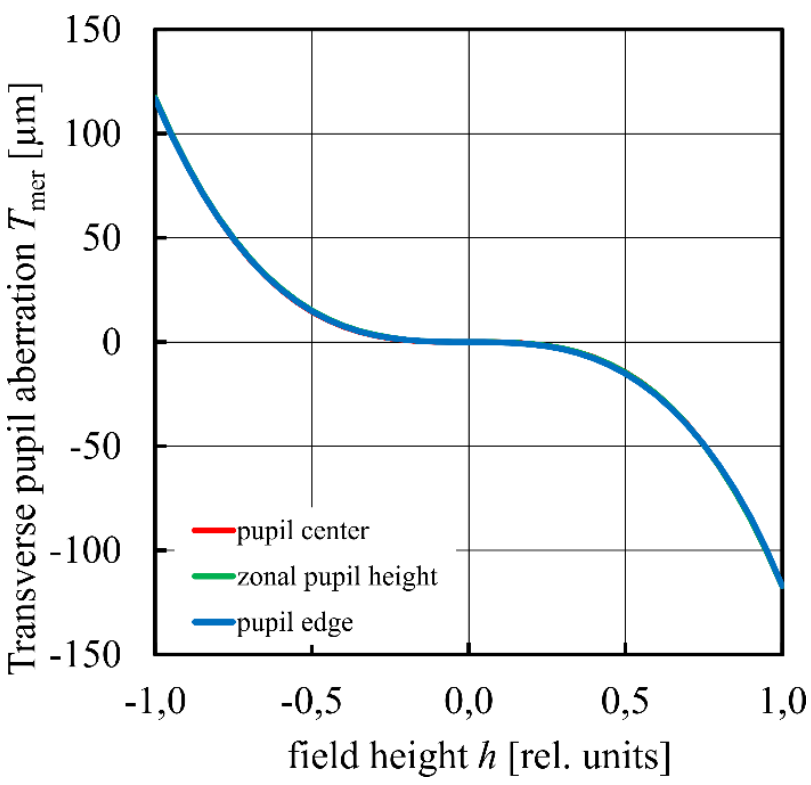

Fig. 4. Transverse pupil aberration $T_{\mathrm{mer}}$ in meridional direction for three resulting pupil heights, that refer to ray angles in object space, namely $0^{\circ}$ (pupil center, red), $8,16^{\circ}$ (zonal, green) and $11,54^{\circ}$ (pupil edge, blue).

Using the results of Table 1, specifically the third line of the last column, one can now estimate the amount of residual induced aberration, i.e. field-cubic coma:

$$
\begin{aligned}
\Delta W=4 & \cdot M_{4} \cdot \beta^{3} \cdot \sigma_{1} \cdot h^{3} r^{3} \cos \vartheta \\
& =\underbrace{4 \cdot \frac{M_{\max }}{R_{\max }} \cdot T_{\max }}_{W_{331, \max }} \cdot \frac{h^{3}}{h_{\max }^{3}} \frac{R^{3}}{R_{\max }^{3}} \cos \vartheta
\end{aligned}
$$

where $\beta \cdot r=R$ as well as Eqns. 7 and 8 have been applied. The resulting coma coefficient yields $W_{331, \max }=$ $-0,147 \lambda$. The induced wavefront aberration $\Delta W$ is added to the wavefront aberration of the initial state and 
compared to the actual wavefront aberration of the perturbed system. The results are given in Fig. 5.

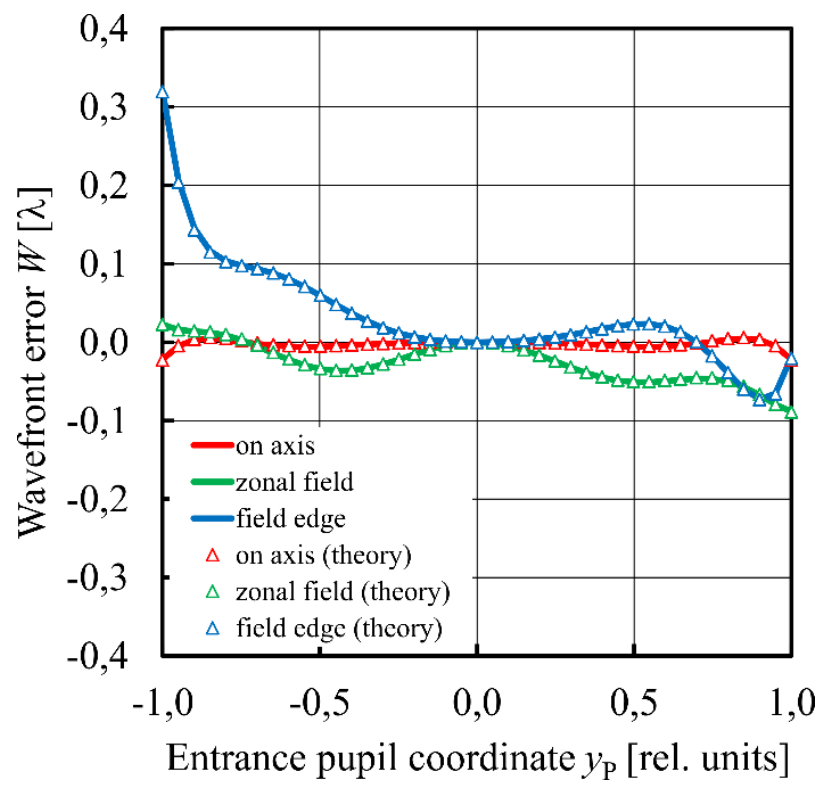

Fig. 5. Wavefront aberration $W$ in meridional direction of the perturbed system given in Fig. 2(c) for three object heights $h=0.0 \mathrm{~mm}$ (on axis, red), $h=3.54 \mathrm{~mm}$ (zonal, green) and $h=5.0 \mathrm{~mm}$ (field edge, blue). Red, green and blue triangles indicate the theoretical prediction of the aberrations according to Eqn. 9.

The thin adaptive phase element has corrected the spherical aberration completely and therefore restored the on-axis performance of the initial system. Indeed, the glass plate itself also causes a small amount of about $0,08 \lambda$ of $5^{\text {th }}$-order spherical aberration. In the given example, this is however compensated by induced $5^{\text {th }}$-order spherical aberration according to Table 1 because of the residual pupil distortion mentioned above. Therefore, a purely $4^{\text {th }}$-order phase correction is sufficient here.

According to the cubic field dependency of the induced coma aberration, no further influence is predicted for the on-axis object point. The zonal object point is only minor affected by the pupil aberration effect. However, the performance at the field edge is significantly changed. As can be seen, the theoretical estimation is in perfect agreement with the actual aberrations determined by optical design software.

\section{Comments and Remarks}

Pupil magnification deviations or pupil distortion both do not cause field-dependent aberrations according to Table 1. If the adaptive element provides enough degrees of freedom, the effects of these aberrations can therefore be compensated by the element itself. Hence, the specific kind of reference or ideal distortion plays a minor role.

First-order deviations, i.e. pupil defocus or pupil magnification deviations, are typically corrected for the reference wavelength in monochromatic applications. For broadband adaptive optical systems, however, they may play a significant role if axial and lateral color are not corrected for the pupil image. In such case, the respective aberrations of Table 1 are then induced for wavelengths other than the reference wavelength, and color aberrations arise. The series expansions of Eqn. 2, 3 and 6 can, of course, be extended also to higher orders. If transverse pupil aberrations are large, e.g, also the $2^{\text {nd }}$-order term of the Tayler series of $M$ is required. For reasons of simplicity, this work only gives the lowest-order results, which have the highest practical relevance.

In the theoretical deviations, the adaptive element is assumed to be infinitely thin. In practical cases, this is unlikely to be true. As a consequence, the element itself may cause field-dependent aberrations once a specific phase is applied. If, e.g, a liquid lens is used for a parabolic or spherical phase, such lens will also intrinsically contribute to the Petzval field curvature. Other field-dependent aberrations may arise if an adaptive element, typically a reflective one, is used in an off-axis setup. These aberrations will then also be induced for perfectly-corrected conjugated pupil images and are therefore not the scope of this work. They may, however, occur in the same order of magnitude as the aberrations given in Table 1.

\section{Conclusion}

Adaptive elements used for wavefront manipulations are typically placed in a conjugated pupil plane, which may suffer from pupil aberrations. Depending on the actual shape of the phase profile, specific types of fielddepending aberrations are induced. This work gives a systematic derivation of the resulting types of induced aberrations for the case of rotationally-symmetric wavefront corrections together with a quantitative estimation of the respective amount of aberration. An example optical system showed perfect agreement with the theoretical prediction. The theory allows for specifying the required pupil correction for a given wavefront manipulation task.

\section{Acknowledgments}

The optical systems have been designed and analyzed with the ZEISS in-house optical design software.

\section{References}

1. J. Sasian, Proc. SPIE 6342, 634208 (2006)

2. M. J. Kidger, Fundamental Optical Design (SPIE Press, Bellingham, 2002)

3. D. Reshidko, J. Sasian, Opt. Eng. 55, 115105 (2016)

4. T. Smith, Trans. Opt. Soc. 23, 139-153 (1921)

5. C. G. Wynne, Proc. Phys. Soc. B 65, 429 (1952)

6. C.-H. Lin et al., J. Opt. 14, 065401 (2012)

7. B. J. Bauman, Proc. SPIE 5903, 59030R (2005)

8. D.L. Fried, J. Opt. Soc. Am. A, 69, 399-406 (1982)

9. R. Kingslake, R. B. Johnson, Lens Design Fundamentals (SPIE Press, Bellingham, 2010) 